\title{
A LiE Subroutine for Computing Prehomogeneous Spaces Associated with Complex Nilpotent Orbits
}

\author{
Steven Glenn Jackson and Alfred G. Noël \\ Department of Mathematics, \\ University of Massachusetts, \\ Boston, MA 02125-3393, USA \\ \{jackson, anoel\}@math.umb.edu
}

\begin{abstract}
We develop a LiE subroutine to compute the irreducible components of certain prehomogeneous spaces that are associated with complex nilpotent orbits. An understanding of these spaces is necessary for solving more general problems in the theory of nilpotent orbits and the representation theory of Lie groups. The output is a set of $\mathrm{LT}_{\mathrm{E}} \mathrm{X}$ statements that can be compiled in a $\mathrm{IAT}_{\mathrm{E}} \mathrm{X}$ environment in order to produce tables. Although the algorithm is used to solve the problem in the case of exceptional complex reductive Lie groups 2, it does describe these prehomogeneous spaces for the classical cases also. Complete tables for the exceptional groups can be found at http://www.math.umb.edu/ anoel/publications/tables/.
\end{abstract}

\section{Introduction}

It has been long established that the representation theory of Lie groups is of fundamental importance to physics, chemistry and mathematics. However it is less known to scientists that this theory is currently being applied in many engineering applications, in computer science and in the development of financial mathematics. The theory is still being developed by mathematicians who are more and more participating in interdisciplinary projects. Consequently, progress in the representation theory of groups should be of interest to scientists and engineers working at the cutting edges of their fields.

The representation theory of semisimple Lie groups (and general reductive Lie groups) is especially important and challenging. Mathematicians have developed a large number of techniques to create different representations of a given Lie group. An understanding of the nilpotent orbits of such groups would provide important information about their possible representations. In the study and classification of such orbits the theory of prehomogeneous spaces plays an important role.

Definition 1 (M. Sato [5]). Let $G$ be a connected semisimple complex Lie group, $V$ a finite dimensional vector space over $\mathbb{C}$ and $\rho$ a rational representation of $G$ in $V$. Then the triple $(G, \rho, V)$ is called a prehomogeneous vector space if 
$G$ admits a Zariski open dense orbit $\Omega$ in $V$. If $\rho$ is irreducible then $(G, \rho, V)$ is said to be irreducible.

We often write $(G, V)$ instead of $(G, \rho, V)$ and $g x$ instead of $(\rho(g))(x)$.

Let $\mathrm{g}$ be a semisimple complex Lie algebra and $G$ its adjoint group. It is a fact that the number of nilpotent orbits of $G$ in $g$ is finite. Let $X$ be a representative of a nilpotent orbit $\mathcal{O}$ of $G$ in $g$. Then from the Jacobson-Morozov theorem $X$ can be embedded into a triple $(H, X, Y)$ with $H$ semisimple and $Y$ nilpotent such that

$$
[H, X]=2 X, \quad[H, Y]=-2 Y, \quad[X, Y]=H .
$$

Such an $(H, X, Y)$ is called an $\mathrm{sl}_{2}$-triple. Given such a triple, the action of $\operatorname{ad}_{H}$ determines a grading $\mathrm{g}=\bigoplus_{i \in Z Z} \mathrm{~g}_{i}$, where $\mathrm{g}_{i}=\{Z \in \mathrm{g}:[H, Z]=i Z\}$.

It is a fact that $\mathrm{g}_{0}$ is a reductive Lie subalgebra of $\mathrm{g}$. Let $G_{0}$ be the connected subgroup of $G$ such that $\operatorname{Lie}\left(G_{0}\right)=\mathrm{g}_{0}$. In 1959, Kostant 4 showed that $\left(G_{0}, \mathrm{~g}_{2}\right)$ is a prehomogeneous vector space. Later, Vinberg [7] generalized Kostant's result by showing that all $\left(G_{0}, g_{i}\right)$ pairs are prehomogeneous vector spaces.

\section{Our goal is to describe the irreducible components of the $G_{0}$-modules $g_{i}$ for all nilpotent orbits of the Lie group $G$ in $g$.}

It is enough to consider the simple complex Lie algebras. We have developed a general theory for classical complex simple Lie groups by exploiting the fact that their nilpotent orbits are parametrized by certain partitions of an integer related to their rank. Such parametrization is not available for exceptional groups. As a result our subroutine is needed in order to solve the problem in the cases where $G$ is of exceptional type. As a byproduct we obtain another method of computing these prehomogeneous spaces for any fixed simple complex classical Lie group. All these results are found in [2].

In order to give the reader the flavor of the general result we provide a complete description of the result in the case of the general linear group.

\section{Type $G L_{n}$}

Let $G L_{n}$ be the set of $n \times n$ complex invertible matrices. Then $\mathrm{g}=\mathrm{gl}_{n}$, the set of all $n \times n$ complex matrices. It is known that the nilpotent orbits of the group $G=G L_{n}$ on $\mathrm{g}$ are parametrized by partitions of $n$. Therefore, if $X$ is a nilpotent element of $\mathrm{g}$ then up to conjugation we can take $\mathrm{X}$ as sum of Jordan blocks of size $d_{i}$ where the $d_{i}$ 's are parts of the partition $\mathbf{d}=\left[d_{1}, \ldots, d_{k}\right]$ representing the class of $X$. For example if $n=8$ and $\mathbf{d}=[3,3,2]$ then

$$
X=\left(\begin{array}{lll|lll|ll}
0 & 1 & 0 & 0 & 0 & 0 & 0 & 0 \\
0 & 0 & 1 & 0 & 0 & 0 & 0 & 0 \\
0 & 0 & 0 & 0 & 0 & 0 & 0 & 0 \\
\hline 0 & 0 & 0 & 0 & 1 & 0 & 0 & 0 \\
0 & 0 & 0 & 0 & 0 & 1 & 0 & 0 \\
0 & 0 & 0 & 0 & 0 & 0 & 0 & 0 \\
\hline 0 & 0 & 0 & 0 & 0 & 0 & 0 & 1 \\
0 & 0 & 0 & 0 & 0 & 0 & 0 & 0
\end{array}\right) .
$$


The Jacobson-Morozov theorem tells us that we can embed $X$ in a triple $(H, X, Y)$. In this case we can choose $H$ to be the following diagonal matrix of trace zero:

$$
H=\operatorname{diag}\left(d_{1}-1, d_{1}-3, \ldots,-d_{1}+3,-d_{1}+1, \ldots, d_{k}-1, \ldots,-d_{k}+1\right)
$$

Let $V=\mathbb{C}^{n}$ be the standard representation of $\mathrm{g}$. Since $H$ is semisimple we must have $V=\bigoplus_{i \in Z} V_{i}$, where $V_{i}$ is the $H$-eigenspace of eigenvalue $i$.

Theorem 1. Maintaining the above notation, $G_{0} \simeq \prod G L\left(V_{i}\right)$ and each $G_{0}$ module $\mathrm{g}_{l} \simeq \bigoplus_{i-j=l} V_{i} \otimes V_{j}^{*}$.

Proof. Let $G^{H}$ be the centralizer of $H$ in $G$. Then $G^{H}$ preserves each eigenspace $V_{i}$ of $H$. Hence $G^{H} \simeq \prod G L\left(V_{i}\right)$. But under the adjoint action $G_{0}=G^{H}$. Let $\mathrm{g}_{0} \simeq \bigoplus \mathrm{gl}\left(V_{i}\right)$ be the Lie algebra of $G_{0}$; then as a $\mathrm{g}_{0}$-module we can identify $\mathrm{g}$ with $V \otimes V^{*}$, which is equivalent to $\bigoplus_{i, j} V_{i} \otimes V_{j}^{*}$. Moreover, as $\mathrm{g}_{0}$-module, $\mathrm{g} \simeq$ $\bigoplus \mathrm{g}_{l}$. It follows that each $\mathrm{g}_{0}$-module $\mathrm{g}_{l}$ is identified exactly with $\bigoplus_{i-j=l} V_{i} \otimes V_{j}^{*}$ since $\operatorname{ad}_{H}$ is a derivation.

Here is an example. Let $X$ be the representative of $\mathbf{d}=[3,3,2]$ given above. Then

$$
H=\operatorname{diag}(2,0,-2,2,0,-2,1,-1) .
$$

Since $\operatorname{dim} V_{2}=\operatorname{dim} V_{0}=\operatorname{dim} V_{-2}=2$ and $\operatorname{dim} V_{1}=\operatorname{dim} V_{-1}=1$ we have

$$
G_{0}=G L_{2} \times G L_{2} \times G L_{2} \times G L_{1} \times G L_{1}
$$

while

$$
\begin{aligned}
& \mathrm{g}_{1} \simeq\left(V_{2} \otimes V_{1}^{*}\right) \oplus\left(V_{1} \otimes V_{0}^{*}\right) \oplus\left(V_{0} \otimes V_{-1}^{*}\right) \oplus\left(V_{-1} \otimes V_{-2}^{*}\right), \\
& \mathrm{g}_{2} \simeq\left(V_{2} \otimes V_{0}^{*}\right) \oplus\left(V_{1} \otimes V_{-1}^{*}\right) \oplus\left(V_{0} \otimes V_{-2}^{*}\right), \\
& \mathrm{g}_{3} \simeq\left(V_{2} \otimes V_{1}^{*}\right) \oplus\left(V_{1} \otimes V_{-2}^{*}\right) \\
& \mathrm{g}_{4} \simeq\left(V_{2} \otimes V_{-2}^{*}\right) .
\end{aligned}
$$

\section{Description of the Algorithm}

Maintaining the above notations, let $\Delta=\left\{\alpha_{1}, \ldots, \alpha_{l}\right\}$ be the Bourbaki set of simple roots of $\mathrm{g}$ and $R^{+}$the positive root system generated by $\Delta$. Then there is a one-to-one correspondence between nilpotent orbits of $G$ in $g$ and a subset of Dynkin diagrams whose nodes are labeled with elements of the set $\{0,1,2\}$. This is the Dynkin-Kostant classification [4. Algorithms for labeling the Dynkin diagrams are found in [1. For example, if $\mathrm{g}=\mathrm{sl}_{8}$ (the set of $8 \times 8$ matrices of trace 0 ) and $\mathcal{O}$ is the nilpotent orbit corresponding to the partition $\mathbf{d}=[3,3,2]$ then $\mathcal{O}$ is associated with the following diagram:

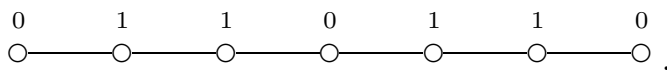


The labels represent the evaluations of the simple roots $\alpha_{i}$ on the diagonal matrix:

$$
\hat{H}=\operatorname{diag}(2,2,1,0,0,-1,-2,-2) .
$$

with $\alpha_{i}(\hat{H})=\hat{h}_{i, i}-\hat{h}_{i+1, i+1}$. Moreover $H$ is conjugate to $\hat{H}$ under the Weyl group $S_{8}$ (the symmetric group on eight letters). See [1 for more details. It follows that $\mathrm{g}_{i} \simeq \bigoplus_{\alpha(\hat{H})=i} \mathrm{~g}^{\alpha}$ where $\mathrm{g}^{\alpha}$ is the root space associated with the root $\alpha$.

It is useful to observe that the type of the semisimple part of the reductive Lie group $G_{0}$ can be read from the diagram. It corresponds to the group generated by the roots labeled with zero. In the above example the semisimple part of $G_{0}$ is of type $A_{1} \oplus A_{1} \oplus A_{1}$.

Definition 2. Let $v$ be a non-zero element of the root space $\mathrm{g}^{\lambda} \subseteq \mathrm{g}_{i}$ and $X_{\alpha} a$ non-zero vector in any $\mathrm{g}^{\alpha} \subseteq\left[\mathrm{g}_{0}, \mathrm{~g}_{0}\right]$ with $\alpha>0$. If $\left[X_{\alpha}, v\right]=0$ for all such $X_{\alpha}$ 's then we say that $v$ is a highest weight vector of $G_{0}$ on $\mathrm{g}_{i}$ and we call $\lambda$ a highest weight of $\mathrm{g}_{i}$.

We shall describe an algorithm which computes the highest weights of $\mathrm{g}_{i}$ given a labeling of the Dynkin diagram corresponding to the nilpotent orbit under consideration. The subroutine is written in the language LiE. Readers who are not familiar with LiE may consult [6]. The highest weights will be expressed in terms of the fundamental weights. For the reader's convenience all $\mathrm{LiE}$ generic functions will be written in boldface characters. The subroutine name is irrdmodule(). Its input consist of a labeling string (usually the BalaCarter label attached to the nilpotent orbit), the Dynkin-Kostant labels and the type of the simple group. The subroutine returns a set of $\mathrm{LT}_{\mathrm{E}} \mathrm{X}$ statements that generate a table containing all the highest weights of all non-zero $\mathrm{g}_{i}$ for $i>0$.

\subsection{Algorithm}

irrdmodule(tex bala;vec label; grp g ) =

\{

\# Formatting statements to be used for exceptional groups

\# bala: Bala-Carter notation of the nilpotent orbit

\# label: Dynkin-Kostant label of the nilpotent orbit

\# g: complex simple Lie algebra

grptype $=$ " $;$ if $\mathrm{g}==\mathrm{G} 2$ then grptype $=$ " $\backslash$ gtwo"; $\mathrm{fi}$; if $\mathrm{g}==\mathrm{F} 4$ then

grptype $=$ "\ffour";fi; if $\mathrm{g}==\mathrm{E} 6$ then grptype $=$ " $\backslash$ esix"; fi; if $\mathrm{g}=\mathrm{\text {E}}$ (

then grptype $=$ "\eseven";fi; if $\mathrm{g}==\mathrm{E} 8$ then grptype $=$ "\eeight";fi; printflag $=0$;

setdefault $(\mathrm{g}) ; \mathrm{n}=\mathbf{n} \_$pos_roots; $\mathrm{l}=$ Lie_rank; alpha = pos_roots;

if size(label) $==1$ then

lookfordegree $=0$; modcounter $=\mathrm{n}$;

while modcounter $>0$ do

degree $=\operatorname{null}(\mathrm{n}) ;$ spaceofinterest $=\operatorname{null}(\mathrm{n}, \mathrm{l})$; 
\# Compute degree positive roots

counter $=0$; for $\mathrm{i}=1$ to $\mathrm{n}$ do for $\mathrm{j}=1$ to $\mathrm{l}$ do

degree $[\mathrm{i}]=$ degree $[\mathrm{i}]+\operatorname{label}[\mathrm{j}] *$ alpha $[\mathrm{i}][\mathrm{j}] ; \mathbf{o d}$;

\# Build the $\mathrm{g}_{i}$ vector space specified by lookfordegree

if degree $[\mathrm{i}]==$ lookfordegree then counter $=$ counter +1 ;

spaceofinterest $[$ counter] = alpha[i]; fi;od;

space $=\operatorname{null}($ counter, 1$)$;

for $\mathrm{i}=1$ to counter do space $[\mathrm{i}]=$ spaceofinterest $[\mathrm{i}]$; od;

dimspace $=$ counter;

modcounter $=$ modcounter - dimspace;

if modcounter $<0$ then print("Negative modcounter"); break;fi;

if dimspace $>0 \& \&$ lookfordegree $>0$ then

\# Set up the output $\mathrm{AT}_{\mathrm{E} X} \mathrm{X}$ statement

latexline $=$ " ;

if printflag $==1$ then latexline $=$ " $\backslash$ cline3-5 \&"; fi;

if printflag $==0$ then printflag $=1$;

latexline $=$ " $\backslash$ hline " + bala + " \&" + grptype;

for $\mathrm{i}=1$ to $\mathrm{l}$ do

latexline = latexline + " $\{"+$ label $[\mathrm{i}]+$ " $\} " ;$ od; fi;

latexline = latexline $+" \& "+$ lookfordegree $+" \& "+$ dimspace + "\&\ba";

\# Find all the highest weights of $\mathrm{g}_{i}$

hw = all_one ( dimspace );

for $\mathrm{i}=1$ to 1 do

if label[i] $==0$ then

for $; \mathrm{j}=1$ to dimspace do candidate $=$ alpha $[\mathrm{i}]+$ space $[\mathrm{j}]$;

\# Check if candidate is in space. If yes then it is not a highest weight

for $\mathrm{k}=1$ to dimspace do

if candidate $==$ space $[\mathrm{k}]$ then $\mathrm{hw}[\mathrm{j}]=0$; break;fi; od; od;fi; od;

\# Compute highest weights

numhw $=0$;

for $\mathrm{i}=1$ to dimspace do numhw $=$ numhw + hw $[\mathrm{i}]$; od;

rhighestweights $=\operatorname{null}($ numhw, 1$)$;

counter $=0$;

for $\mathrm{i}=1$ to dimspace do

if hw $[\mathrm{i}]==1$ then

counter $=$ counter +1 ; rhighestweights $[$ counter $]=$ space $[\mathrm{i}] ; \mathbf{f i} ; \mathbf{o d}$;

\# Convert to fundamental weights

if numhw $>0$ then

highestweights $=$ rhighestweights $*$ Cartan;

for $\mathrm{i}=1$ to numhw do latexline = latexline + "(";

for $\mathrm{j}=1$ to $\mathrm{l}$ do 


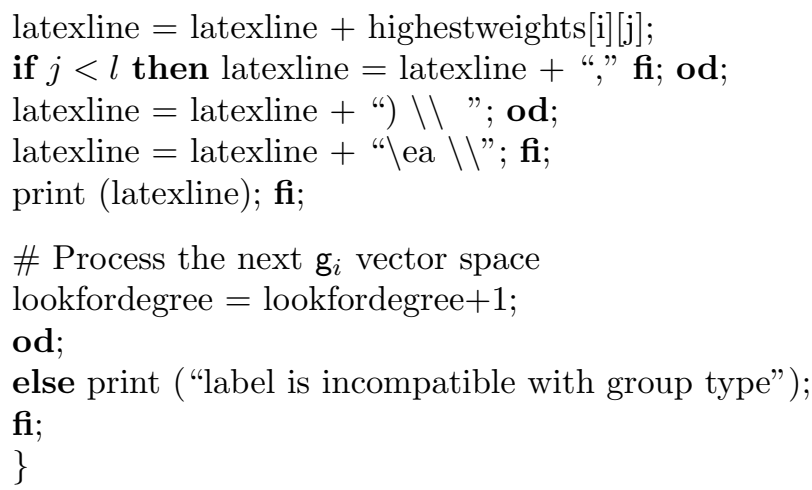

Example 1. We compute the highest weights of each of the four $\mathrm{g}_{i}$ modules associated with the nilpotent orbit $\mathbf{d}=[3,3,2]$ when $\mathrm{g}=\mathrm{sl}_{8}$. Since $\mathrm{g}_{0}$ is reductive we only need to consider the action of its semisimple part $\left[\mathrm{g}_{0}, \mathrm{~g}_{0}\right]$. The subroutine is placed in a file called phmod which has to be loaded in the LiE environment. Here is the LiE session that produces Table 1

LiE version 2.2 created on Nov 221997 at 16:50:29 Authors: Arjeh M. Cohen, Marc van Leeuwen, Bert Lisser. Mac port by S. Grimm Public distribution version

type '?help' for help information

type '?' for a list of help entries.

$>$ read phmod

$>$ irrdmodule( " $2,2,1,0,0,-1,-2,-2 ",[0,1,1,0,1,1,0], A 7)$

Observe that since the number of positive roots of $\mathrm{sl}_{8}$ is 28 and the semisimple part of $\mathrm{g}_{0}$ is of type $A_{1} \oplus A_{1} \oplus A_{1}$ the sum of the dimensions of the $\mathrm{g}_{i}$ 's is correct. By the "Theorem of the Highest Weight" [3-p. 279], $\mathrm{g}_{i}$ is completely determined as $\mathrm{g}_{0}$-module by its highest weights. Moreover, when interpreting the results given in the table, one should be aware that the action of the semisimple part of $\mathrm{g}_{0}$ on $\mathrm{g}_{i}$ is completely determined by those coefficients associated with the nodes of Dynkin-Kostant label 0; the other coefficients affect only the action of the center of $g_{0}$, which in any case must act by scalars on each irreducible component of $\mathrm{g}_{i}$. Let us denote the seven fundamental weights of $\mathrm{sl}_{8}$ by $\omega_{i}$ for $1 \leq i \leq 7$. Then (disregarding the action of the center) we see that

$$
\begin{aligned}
& \mathrm{g}_{1} \simeq V^{\omega_{1}} \oplus V^{\omega_{4}} \oplus V^{\omega_{4}} \oplus V^{\omega_{7}} \\
& \mathrm{~g}_{2} \simeq V^{0} \oplus V^{\omega_{1}+\omega_{4}} \oplus V^{\omega_{4}+\omega_{7}} \\
& \mathrm{~g}_{3} \simeq V^{\omega_{1}} \oplus V^{\omega_{7}} \\
& \mathrm{~g}_{4} \simeq V^{\omega_{1}+\omega_{7}} .
\end{aligned}
$$


Table 1.

\begin{tabular}{|c|c|c|c|c|}
\hline \multicolumn{5}{|c|}{ Example in $\mathrm{sl}_{8}$ revisited } \\
\hline Elementary divisors & Label & i & $\operatorname{dim}_{i}$ & Highest weights of $g_{i}$ \\
\hline \multirow[t]{4}{*}{$2,2,1,0,0,-1,-2,-2$} & \multirow[t]{4}{*}{0110110} & 1 & 8 & $\begin{array}{l}(1,1,-1,0,0,0,0) \\
(0,-1,1,1,-1,0,0) \\
(0,0,-1,1,1,-1,0) \\
(0,0,0,0,-1,1,1)\end{array}$ \\
\hline & & 2 & 9 & $\begin{array}{l}(0,-1,1,0,1,-1,0) \\
(1,0,0,1,-1,0,0) \\
(0,0,-1,1,0,0,1)\end{array}$ \\
\hline & & 3 & 4 & $\begin{array}{l}(1,0,0,0,1,-1,0) \\
(0,-1,1,0,0,0,1)\end{array}$ \\
\hline & & 4 & 4 & $(1,0,0,0,0,0,1)$ \\
\hline
\end{tabular}

where $V^{\lambda}$ denotes the irreducible $\left[\mathrm{g}_{0}, \mathrm{~g}_{0}\right]$-module of highest weight $\lambda$. We note that since $\mathrm{g}_{-i}$ is dual to $\mathrm{g}_{i}$, the module structure of $\mathrm{g}_{-i}$ is easily determined from that of $\mathrm{g}_{i}$.

The correctness of the algorithm should be evident to anyone familiar with the representation theory of complex reductive Lie groups. The computation of highest weights is accomplished by finding the set of vectors in each $g_{i}$ which are annihilated by all positive simple root spaces in $\left[\mathrm{g}_{0}, \mathrm{~g}_{0}\right]$. Such root spaces correspond to the simple roots of Dynkin-Kostant label 0. Since go acts by the adjoint action, the subroutine returns only the roots $\beta$ such that $\mathrm{g}^{\beta}$ lies in $\mathrm{g}_{i}$ and $\left[X_{\alpha}, X_{\beta}\right]=0$ for all positive $\alpha$ with $\mathrm{g}^{\alpha} \subset\left[\mathrm{g}_{0}, \mathrm{~g}_{0}\right]$. This is exactly the set of highest weights of $g_{i}$, which is recorded in the array rhighestweights. Finally, we express the highest weights in terms of the fundamental weights using the Cartan matrix of $\mathrm{g}$.

A worst case analysis of the code reveals the algorithm is $\mathcal{O}(\operatorname{rank}(\mathrm{g}) \times$ $\left.\left(n \_ \text {pos_roots }\right)^{3}\right)$. This is due to the fact that the control variable of the outer while loop, modcounter, is bounded by the number of positive roots and that the more intensive internal for loop is at worst executed $\left(\operatorname{rank}(\mathrm{g}) \times\left(n_{-} \text {pos_roots }\right)^{2}\right)$ times. Of course we are assuming that the $\mathrm{LiE}$ internal functions are very fast. From our experience we believe that they are optimal. On average the subroutine performs very well. We use it to compute the irreducible modules of the prehomogeneous spaces associated to all the nilpotent orbits of the exceptional complex simple groups. Complete tables for the exceptional groups can be found at http://www.math.umb.edu/ anoel/publications/tables/. For more information see 2]. The computations were carried on an IMac G4 with speed $1 \mathrm{GHz}$ and $1 \mathrm{~Gb}$ SDRAM of memory.

\section{Conclusion and Future Work}

We presented a LiE implementation of a simple algorithm for computing the module structures of a large class of prehomogeneous spaces, namely those asso- 
ciated with nilpotent orbits in the adjoint representations of complex reductive Lie groups. In [2] we have developed general methods for the classical types, taking advantage of the parametrization of the nilpotent orbits by partitions. We developed the algorithm presented here to solve the problem for groups of exceptional type. The algorithm as implemented in LiE is stable and of a low complexity. Our implementation is clear, which makes analysis very easy. The correctness of the algorithm is a consequence of the well-known "Theorem of the Highest Weight." Several other authors have treated special cases of this problem; our results generalize theirs to a complete solution for complex reductive groups.

We are currently working on extending these results to non-compact real reductive Lie groups. In light of the Kostant-Sekiguchi correspondence, it suffices to consider the action of the subgroup $K_{\mathbb{C}} \subset G_{\mathbb{C}}$ whose real form is maximal compact in $G$ on the complex symmetric space $G_{\mathbb{C}} / K_{\mathbb{C}}$. This work has already started and we have obtained preliminary results.

\section{References}

1. Collingwood, D. H. and McGovern, W. M. Nilpotent orbits in semisimple Lie algebras. Van Nostrand Reinhold Mathematics Series, New York (1992).

2. Jackson, S. G. and Noël, A. G. Prehomogeneous spaces associated with complex nilpotent orbits. To appear in Journal of Algebra.

3. Knapp, A. W. Lie groups beyond an introduction. Second edition, Birkhaüser Progress in Mathematics 140 (2002)

4. Kostant, B. The principal three dimensional subgroup and the Betti numbers of a complex Lie group. Amer. J. Math., Vol. 81, (1959) 973-1032.

5. Sato, M. and Kimura, T. A classification of irreducible prehomogeneous vector spaces and their relative invariants. Nagoya Math. J. 65 (1977), 1-155.

6. Van Leeuwen, M. A. A., Cohen, A. M., and Lisser, B. LiE: A package for Lie group computations, Computer Algebra Nederland, Amsterdam, Netherlands (1992)

7. Vinberg, E. B. On the classification of the nilpotent elements of graded Lie algebras, Doklady Academii Nauk SSSR 225 (1975b), 745-748 (Russian). English translation: Soviet Math. Doklady 16 (1975), 1517 - 1520. 